\title{
Ictiofauna associada às raízes de mangue do estuário do Rio Pacoti - CE, Brasil
}

\author{
Frederico Moreira Osório ${ }^{1,2,3}$, Wander Oliveira Godinho ${ }^{I}$ \& Tito Monteiro da Cruz Lotufo ${ }^{I}$ \\ ${ }^{1}$ Laboratório de Ecologia Animal, Instituto de Ciências do Mar, Universidade Federal do Ceará - UFC, \\ Av. Abolição, n. 3207, Meireles, Fortaleza, CE, Brasil \\ ${ }^{2}$ Instituto Brasileiro do Meio Ambiente e dos Recursos Naturais Renováveis - IBAMA/SUPES/RN, \\ Av. Alexandrino de Alencar, n. 1399, Tirol, Natal, RN, Brasil \\ ${ }^{3}$ Autor para correspondência: Frederico Moreira Osório, e-mail: fredosorio@gmail.com
}

OSÓRIO, F.M., GODINHO, W.O. \& LOTUFO, T.M.C. Fish fauna associated to mangrove roots at the Pacoti River estuary. Biota Neotrop. 11(1): http://www.biotaneotropica.org.br/v11n1/en/abstract?shortcommunication+bn00711012011.

\begin{abstract}
The mangrove vegetation along the margins of estuaries is an important habitat for fish, serving as feeding, shelter and nursery zones. The present work describes the fish fauna associated to mangrove roots at the Pacoti River estuary. A total of ten visual censuses of 90 minutes each were conducted, yielding the number of 671 fish counted, with an average of 0.74 counts/minute. The fishes were identified pertaining to 22 species from 18 different families. All the studied species are originally marine, although the samples were mainly carried out during the rainy season. Gerreidae and Lutjanidae were the more representative families. The species Lutjanus alexandrei, Lutjanus jocu and Bathygobius soporator were present in all censuses and the first two were also the most abundant, with 48 and $17 \%$ of the total counts, respectively. Taking into account size estimations of the fish, it was verified that mangrove roots are inhabited mainly by juveniles of both species, indicating the use of this habitat as a nursery area. However, in the least complex sampling site, specimens of genus Lutjanus were sought in higher quantities, comprinsing all size classes, thus suggesting the abundance of roots in Pacoti River might not influence the abundance of sheltered fishes. Another important observation is that almost $70 \%$ of the species present in the mangrove roots are typical of reef habitats, indicating the existence of strong connections between these two ecosystems. The results emphasize the importance of this habitat for ichthyofauna conservation to coastal areas. In addition, studies regarding to fish recruitment, reproductive and trophic patterns are needed, especially to those two main important species described herein.
\end{abstract}

Keywords: estuarine fishes, nursery, Lutjanus, visual census.

OSÓRIO, F.M., GODINHO, W.O. \& LOTUFO, T.M.C. Ictiofauna associada às raízes de mangue do Estuário do Rio Pacoti - CE, Brasil. Biota Neotrop. 11(1): http://www.biotaneotropica.org.br/v11n1/pt/abstract?shortcommunication+bn00711012011.

Resumo: Margens de estuários com a vegetação de manguezal formam um importante hábitat para peixes, pois são zonas de alimentação, abrigo e berçário. O presente trabalho tem como objetivo caracterizar a ictiofauna associada às raízes de mangue do estuário do Rio Pacoti (CE). Foram realizados 10 censos visuais com duração de 90 minutos cada. Foram feitas 671 avistagens, com densidade média de 0,74 avistagens/minuto. Os indivíduos pertencem a 22 espécies e 18 famílias. Todas as espécies registradas são de origem marinha, apesar das amostragens serem realizadas principalmente no período chuvoso. As famílias que apresentaram um maior número de espécies foram Gerreidae e Lutjanidae. As espécies que apresentaram maior frequência de ocorrência foram Lutjanus alexandrei, Lutjanus jocu e Bathygobius soporator, estando presentes em todos os censos. As duas espécies mais abundantes foram L. alexandrei (48\%) e L. jocu (17\%). Levando-se em consideração as estimativas de tamanho destas espécies, foi verificado que as raízes de mangue são habitadas principalmente por indivíduos jovens de ambas as espécies, o que leva a supor que estas utilizam tal habitat como área de berçário. Contudo, na estação de coleta com menor complexidade estrutural foi avistado o maior número de indivíduos do gênero Lutjanus, em praticamente todas as faixas de comprimento, o que sugere que no Rio Pacoti a quantidade de raízes pode não influenciar na quantidade de indivíduos abrigados. Aproximadamente $70 \%$ das espécies avistadas também ocorrem em ambientes recifais, o que demonstra a existência de fortes conexões entre esses dois ecossistemas. Os resultados encontrados enfatizam a importância do habitat estudado para a conservação da ictiofauna costeira e apontam a necessidade de pesquisas relacionadas principalmente ao recrutamento, padrões reprodutivos e tróficos das duas espécies mais representativas.

Palavras-chave: peixes estuarinos, berçário, Lutjanus, censo visual. 


\section{Introdução}

Os estuários funcionam como áreas de alimentação e refúgio para juvenis de peixes marinhos que se reproduzem no mar. Além disso, tais ecossistemas compreendem áreas de alimentação utilizadas sazonalmente por adultos de peixes marinhos, além de servirem como habitat definitivo para outras espécies (Lowe-MacConnell 1999). As margens de estuários com a vegetação de manguezal formam um importante hábitat para peixes, pois são zonas de alimentação, abrigo e berçário (Thayer et al. 1987, Vance et al. 1996, Ley et al. 1999, Morton 1990, Nagelkerken et al. 2000, Nakamura et al. 2008).

A diminuição significativa das áreas de manguezal e a degradação de importantes complexos estuarinos e baías vêm reduzindo o hábitat de muitas populações de animais e implicando num aumento da competição por alimento, contribuindo consideravelmente para a aceleração da mortalidade de espécies essenciais para a subsistência de comunidades tradicionais e para a perda da biodiversidade (Campos et al. 2003). Diegues (1987) aponta que áreas remanescentes de manguezais ainda são utilizadas por comunidades de pescadores e extrativistas, que delas dependem para a sua sobrevivência.

A ictiofauna das áreas de manguezal já foi estudada em algumas localidades do nordeste brasileiro. Eskinazi (1970, 1972), Vasconcelos-Filho \& Oliveira (1999) e Paiva et al. (2008) publicaram trabalhos sobre a ictiofauna de alguns manguezais de Pernambuco. Oliveira $(1972,1974,1976)$ e Araújo et al. (2000, 2004) realizaram inventários sobre a ictiofauna estuarina no Ceará utilizando coletas com redes e dados disponíveis na literatura. Apenas Freitas et al. (2006) realizaram censos visuais em um manguezal com o propósito de verificar a função das mesas de cultivo de ostras como atratores para peixes. Contudo, não foram realizadas pesquisas no nordeste brasileiro que visassem estudar a comunidade de peixes que habitam as estruturas formadas pelas raízes de mangues em estuários utilizando observação subaquática.

Este trabalho tem como objetivo inventariar a ictiofauna que ocupa as raízes de mangue do estuário do Rio Pacoti no Ceará por meio de observações subaquáticas.

\section{Material e Métodos}

O presente estudo foi realizado no Rio Pacoti, que possui aproximadamente $150 \mathrm{~km}$ de extensão, tendo sua nascente no maciço de Baturité e desembocadura na região metropolitana de Fortaleza. O estuário deste rio possui uma área de $141 \mathrm{~km}^{2}$, descarga fluvial de $19 \mathrm{~m}^{3} / \mathrm{s}$, volume de $518,502 \mathrm{~m}^{3}$ e prisma de maré de $476,011 \mathrm{~m}^{3}$ (ZEE 2005a). A vegetação predominante no estuário consiste de Rhizophora mangle, Avicennia schaueriana, Avicennia germinans, Laguncularia racemosa e Cornocarpus erectus (Oliveira et al. 1988).

Para orientar a escolha das três estações de amostragem, foram realizadas quatro visitas ao estuário e quatro censos experimentais. A estação 1 fica localizada a aproximadamente $1.150 \mathrm{~m}$ da desembocadura do rio, dista cerca de $2.500 \mathrm{~m}$ da estação 2. As estações 2 e 3 estão separadas por 833 m (Figura 1). As estações 1 e 2 apresentam uma grande quantidade de raízes-escora da espécie Rhizophora mangle, incrustada por Crassostrea rhizophorae. A estação 3 apresenta principalmente raízes de árvores mortas, ou de pequeno porte, das espécies de Avicennia e Laguncularia racemosa, além de trechos em início de colonização por $R$. mangle. As duas primeiras estações mostram maior complexidade estrutural que a terceira estação devido à maior quantidade de raízes, galhos e fauna incrustante.

As coletas de dados foram realizadas por meio de 10 censos visuais, nas margens do rio, que foram percorridas durante a preamar (Tabela 1). Essas condições permitem que o mergulhador realize as observações nas raízes e nos galhos de manguezal. Todos os indivíduos avistados entre ou sobre as raízes e galhos eram identificados, contados e tinham os seus tamanhos estimados. O deslocamento unidirecional realizado pelo amostrador visou diminuir o risco de recontagem de animais. O tempo de duração de cada censo foi estabelecido a partir de uma amostragem piloto de 120 minutos, que indicou como ideal a duração de 90 minutos, pois poucas espécies foram acrescidas após esse tempo. Foi realizado um total de 900 minutos de observação (15 horas). A estimativa de comprimento dos indivíduos foi realizada em cinco censos e compreendia as seguintes classes, em centímetros, nos seguintes intervalos: $<5$; $5-10 ; 10-15 ; 15-20 ;>20$. A salinidade da água em cada censo foi obtida com auxílio de refratômetro. A visibilidade foi estimada através da distância máxima de identificação subaquática de um objeto retangular de cor branca com área de $600 \mathrm{~cm}^{2}(30 \times 20 \mathrm{~cm})$. Os dados foram anotados em uma prancheta de PVC e posteriormente transferidos para planilhas eletrônicas.

As espécies foram classificadas em residentes, marinhas dependentes e marinhas visitantes, de acordo com Freitas et al.(2006). O componente residente foi composto por espécies que passam todo o ciclo de vida nas águas estuarinas, podendo ser encontradas também em habitat marinho costeiro, ou na água doce. O componente marinho dependente incluiu as espécies de origem marinha que obrigatoriamente utilizam as águas estuarinas, seja para alimentação, ou para completar parte do seu ciclo reprodutivo. O componente marinho visitante abrangeu as espécies de origem marinha que passam a maior parte do ciclo de vida no mar, onde desovam, penetrando nas águas estuarinas em períodos regulares ou ocasionalmente, sem aparente exigência estuarina.

Todas as amostragens foram realizadas na estação chuvosa (Tabela 1). A falta de regularidade das amostragens pode ter comprometido os resultados devido a possíveis sazonalidades de ocorrência de algumas espécies.

\section{Resultados e Discussão}

Ao todo foram realizadas 671 avistagens, perfazendo uma densidade média de 0,74 registros/minuto. Os indivíduos pertenciam a 22 espécies de 18 famílias (Tabela 2). As famílias que apresentaram um maior número de espécies foram Gerreidae e Lutjanidae. Devido a necessidade de verificação de caracteres merísticos as famílias Centropomidae, Gerreidae, Mugilidae e Paralichthyidae foram identificados ao nível de família e gênero. As famílias Blenniidae e Scaridae foram identificados ao nível de família e gênero devido a dificuldade de identificação visual de jovens.

Tabela 1. Informações sobre o número do censo, estação, data, horário e nível de maré no início das amostragens.

Table 1. Information about number of the census, station, date, time, and tidal level at the beginning of the samples.

\begin{tabular}{ccccc}
\hline Censo & Estação & Data & $\begin{array}{c}\text { Horário } \\
\text { (horas) }\end{array}$ & $\begin{array}{c}\text { Nível de maré } \\
(\mathbf{m})\end{array}$ \\
\hline 1 & 1 & $08 / 01 / 2005$ & $15: 30$ & 1.2 \\
2 & 1 & $20 / 01 / 2005$ & $12: 30$ & 1.0 \\
3 & 2 & $28 / 01 / 2005$ & $8: 00$ & 1.5 \\
4 & 2 & $05 / 02 / 2005$ & $15: 45$ & 1.2 \\
5 & 3 & $05 / 03 / 2005$ & $13: 30$ & 1.0 \\
6 & 1 & $29 / 07 / 2005$ & $12: 00$ & 1.0 \\
7 & 3 & $26 / 03 / 2006$ & $15: 00$ & 1.4 \\
8 & 1 & $05 / 01 / 2007$ & $16: 00$ & 1.3 \\
9 & 2 & $29 / 03 / 2007$ & $12: 30$ & 1.2 \\
10 & 3 & $23 / 05 / 2007$ & $08: 30$ & 0.9 \\
\hline
\end{tabular}


Apesar das amostragens terem sido realizadas principalmente no período chuvoso, foi verificado que todas as espécies avistadas são de origem marinha (Tabela 2). Este fato pode estar relacionado às salinidades elevadas encontradas no estuário. A análise da água coletada em cada estação não revelou diferença importante de salinidade no tempo e no espaço, sendo que a mínima e a máxima foram 36 e 40, respectivamente, tais salinidades impossibilitam a presença de espécies fluviais nas estações de coleta, mesmo no período chuvoso. Este resultado sugere que as estações de coleta e épocas em que foram realizadas as amostragens não influenciaram na salinidade a que os peixes são submetidos, de modo que as mesmas espécies devem estar presentes na estação seca. Análises de parâmetros físico-químicos, realizadas no período chuvoso, dos estuários cearenses, sugerem que todos os estuários deste estado são dominados por condições marinhas (ZEE 2005a). As salinidades elevadas ainda que no período chuvoso pode ser resultado do controle artificial da vazão fluvial do Rio Pacoti através de barragens. Esta prática é bastante comum na maioria dos rios cearenses (ZEE 2005b, Lacerda 2006).

A ausência de amostragens na estação seca comprometeu o registro de variações sazonais na ocorrência de espécies e nas abundâncias das espécies encontradas. Outra limitação que deve ser prontamente reconhecida diz respeito à visibilidade. As águas estuarinas são notórias por sua alta produtividade e grande quantidade de material em suspensão (Odum 1988). Apesar da limitação metodológica no registro das espécies em função das baixas condições de visibilidade, a pouca profundidade em que os censos visuais foram realizados permitiu a avistagem dos peixes que se encontravam entre as raízes, sobre as raízes ou em áreas próximas, em toda a coluna d’água.
Dentre as espécies, Lutjanus alexandrei Moura \& Lindeman, 2007, Lutjanus jocu (Bloch \& Schneider, 1801) e Bathygobius soporator (Valenciennes, 1837) foram vistas em todos os censos. Outras espécies frequentemente encontradas foram Hippocampus reidi Ginsburg, 1933 (80\%), Eucinostomus sp. e Sphoeroides testudineus (Linnaeus, 1758) (70\%) e Haemulon parra (Desmarest, 1823), que esteve presente em $60 \%$ dos censos (Tabela 2).

As espécies mais abundantes foram Lutjanus alexandrei (48\%), Lutjanus jocu (17\%) e Eucinostomus sp. (8\%), seguidas de Sphoeroides testudineus (6\%), Hippocampus reidi (4\%), Bathygobius soporator (4\%) e Haemulon parra (4\%). As outras espécies juntas contribuíram com 9\% dos indivíduos avistados (Figura 2). Lutjanus alexandrei e $L$. jocu apresentaram uma baixa frequência relativa nos trabalhos realizados no estuário do Rio Formoso, PE, por Paiva et al. (2008, 2009). Propomos que esta diferença na frequência relativa encontrada entre o presente trabalho e os trabalhos citados pode estar relacionada a metodologia utilizada, o que demonstra a importância de amostragens com utilização de observação subaquáticas em ambientes estuarinos.

Espécies de todas as categorias ecológicas foram avistadas nas três estações de coleta. Provavelmente a pequena distância entre as estações e a semelhança entre as salinidades encontradas contribuíram para este resultado. Contudo, as espécies residentes foram menos representativas em número de espécie e número de indivíduos avistados.

Em número de espécies, as marinhas dependentes e as marinhas visitantes contribuíram cada qual com $42 \%$ do total de espécies (oito espécies cada) e as residentes representaram apenas $16 \%$ das espécies (três espécies) (Tabela 3). Um menor número de espécies residentes

Tabela 2. Lista das famílias, espécies e número de indivíduos (N) observados entre as raízes de mangue ou em áreas próximas, classificações ecológicas das espécies e as suas frequências relativas de ocorrência (\%Fr). As famílias estão listadas em ordem evolutiva segundo Nelson (2006).

Table 2. Family, species, and number of individuals $(\mathrm{N})$ observed in mangrove roots or nearby areas. Ecological group of species and its relative frequency of occurrence (\%Fr). Families are listed following evolutive order, by Nelson (2006).

\begin{tabular}{|c|c|c|c|c|}
\hline Família & Espécie & Classificação ecológica & $\mathbf{N}$ & $\% \mathbf{F r}$ \\
\hline Batrachoididae & Batrachoides surinamensis (Bloch \& Schneider, 1801) & - & 1 & 10 \\
\hline Mugilidae & Mugil sp.* & Marinha dependente ${ }^{1}$ & 3 & 10 \\
\hline Syngnathidae & Hippocampus reidi Ginsburg, $1933 * *$ & Marinha visitante $^{1}$ & 29 & 80 \\
\hline Centropomidae & Centropomus spp. ${ }^{*}$ & Marinha dependente ${ }^{1}$ & 14 & 20 \\
\hline Serranidae & Mycteroperca bonaci (Poey, 1860)* & Marinha visitante $^{2}$ & 1 & 10 \\
\hline \multirow[t]{3}{*}{ Lutjanidae } & Lutjanus alexandrei Moura \& Lindeman, 2007* & Marinha visitante $^{1}$ & 319 & 100 \\
\hline & Lutjanus cyanopterus (Cuvier, 1828) & Marinha dependente ${ }^{3}$ & 2 & 10 \\
\hline & Lutjanus jocu (Bloch \& Schneider, 1801)* & Marinha visitante $^{1}$ & 116 & 100 \\
\hline \multirow[t]{3}{*}{ Gerreidae } & Diapterus sp. ${ }^{*}$ & Marinha dependente ${ }^{1}$ & 6 & 20 \\
\hline & Eucinostomus melanopterus (Bleeker, 1863)* & Marinha dependente ${ }^{1}$ & 12 & 40 \\
\hline & Eucinostomus sp. ${ }^{*}$ & Marinha dependente ${ }^{1}$ & 51 & 70 \\
\hline Haemulidae & Haemulon parra (Desmarest, 1823)* & Marinha visitante $^{1}$ & 27 & 60 \\
\hline Sparidae & Archosargus probatocephalus (Walbaum, 1792)* & Marinha visitante $^{1}$ & 2 & 10 \\
\hline Scaridae & Sparisoma sp. ${ }^{*}$ & Marinha visitante $^{1}$ & 10 & 20 \\
\hline Blenniidae & Indefinida & - & 5 & 30 \\
\hline Gobiidae & Bathygobius soporator (Valenciennes, 1837) & Residente $^{1}$ & 25 & 100 \\
\hline Ephippidae & Chaetodipterus faber (Broussonet, 1782)* & Marinha dependente $^{1}$ & 1 & 10 \\
\hline Acanthuridae & Acanthurus chirurgus (Bloch, 1787)** & Marinha visitante ${ }^{1}$ & 2 & 20 \\
\hline Sphyraenidae & Sphyraena barracuda (Walbaum, 1792)* & Marinha dependente ${ }^{1}$ & 1 & 10 \\
\hline Paralichthyidae & Indefinida & - & 2 & 10 \\
\hline Achiridae & Trinectes paulistanus (Miranda-Ribeiro, 1915) & Residente $^{1}$ & 1 & 10 \\
\hline Tetraodontidae & Sphoeroides testudineus (Linnaeus, 1758) & Residente $^{1}$ & 41 & 70 \\
\hline
\end{tabular}

* Peixes utilizados para consumo; ** peixes utilizados na exportação de ornamentais; ${ }^{1}$ Vasconcelos-Filho \& Oliveira (1999); ${ }^{2}$ Randall (1967) apud

Freitas et al.(2006); ${ }^{3}$ Allen (1985); Moura et al. (2001). 
também foi encontrado por Freitas et al. (2006) realizando censo visual em cultivos de ostras instalados no estuário do Rio Jaguaribe, CE, por Vasconcelos-Filho \& Oliveira (1999) no Canal de Santa Cruz, PE, por Villarroel (1994) em um estuário na Venezuela e por Paiva et al. (2008, 2009 no estuário do Rio Formoso, PE. O menor número de representantes de espécies residentes em relação às espécies migratórias em estuários se deve aos complexos mecanismos fisiológicos necessários à vida em um ambiente caracterizado por consideráveis variações físico-químicas (Albaret et al. 2004, Loebmann \& Vieira 2005).

Em termos do número de avistagens, a categoria mais representativa foi a marinha visitante, $\operatorname{com} 76 \%$, seguida por marinha dependente (14\%) e residente (10\%) (Tabela 3). A maior abundância de peixes marinhos visitantes resulta da grande quantidade de indivíduos de Lutjanus alexandrei avistados.

Levando-se em consideração as estimativas de tamanho das duas espécies mais abundantes, L. alexandrei e $L$. jocu foi possível verificar que as raízes de mangue são habitadas principalmente por indivíduos com comprimentos inferiores a $10 \mathrm{~cm}$, ou seja, indivíduos jovens (Allen 1985, Moura \& Lindeman 2007), o que indica a utilização de tal habitat como área de berçário. Peixes jovens são atraídos para as raízes do manguezal devido a sua complexidade, a qual disponibiliza proteção contra a predação e alimento (Laegdsgaard \& Johnson 2001). Lutjanideos se alimentam principalmente de crustáceos e peixes (Menezes \& Figueiredo 1980), itens encontrados entre as raízes de mangue.

$\mathrm{Na}$ estação com menor complexidade estrutural (Estação 3), foi avistado o maior número de indivíduos do gênero Lutjanus, em praticamente todas as faixas de comprimento (Tabela 4), o que sugere que no Rio Pacoti a quantidade de raízes pode não influenciar na quantidade de indivíduos abrigados. Deste modo, propomos que, provavelmente, a disponibilidade de alimento para as duas espécies mais abundantes de Lutjanidae seja semelhante entre as estações de coleta com diferentes complexidades estruturais, ou esteja disponível em maior quantidade na Estação 3. Propomos também que a proteção contra predadores não é o motivo da ocupação de L. alexandrei e $L$. jocu nestes habitats, já que em todos os census as espécies piscívoras avistadas encontravam-se em estágio juvenil. Sabe-se que o reduzido número de peixes carnívoros de grande porte favorece a abundância de peixes em estuários (Mullin 1995). Por outro lado, devemos levar em consideração que o maior número de raízes serve de obstáculo ao observador, de forma que as avistagens em áreas com alta densidade de raízes podem ser subestimadas.

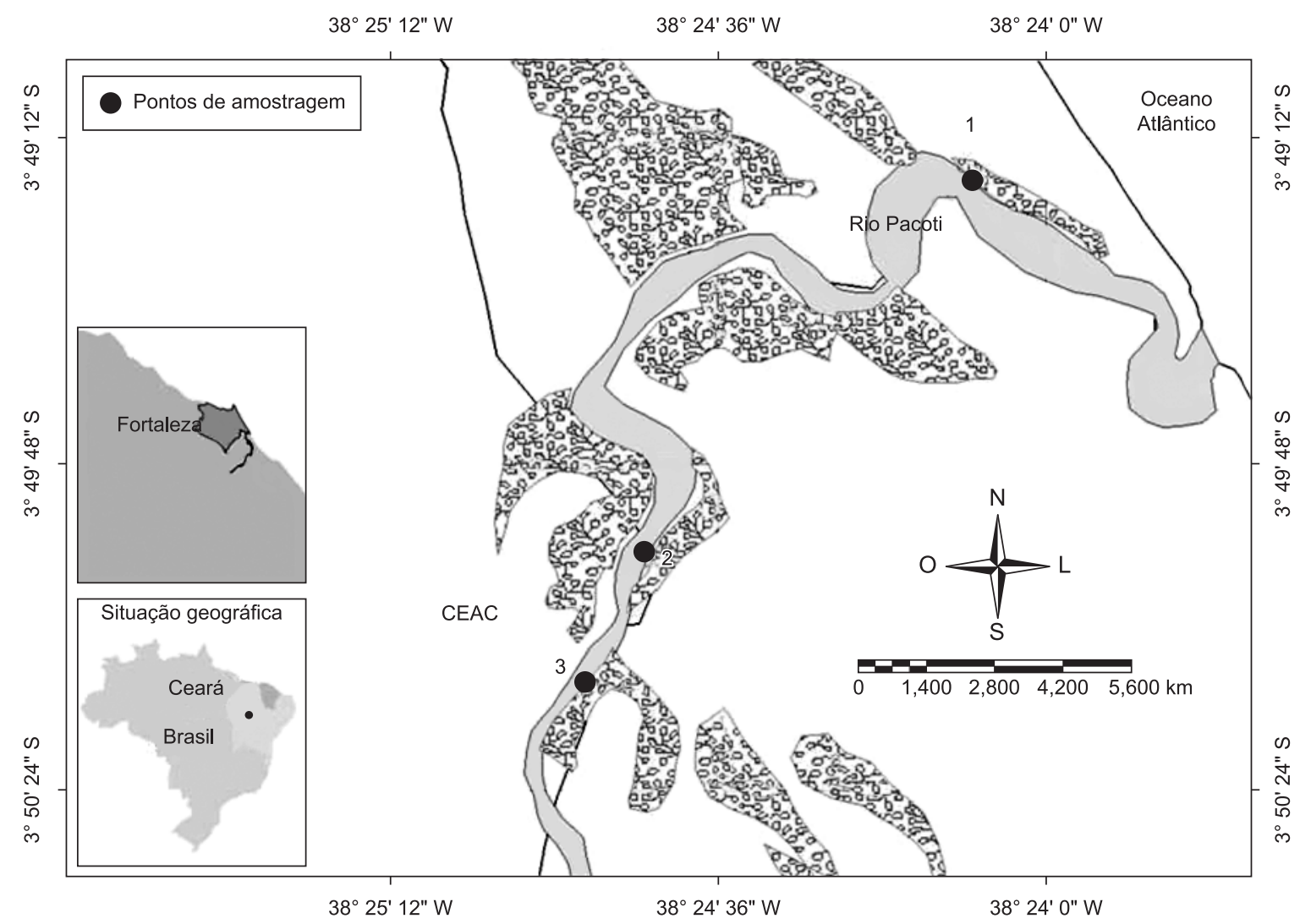

Figura 1. Área de estudo, demonstrando as estações de coleta.

Figure 1. Study site, showing the sampling areas.

Tabela 3. Frequência de ocorrência, contribuição em número de espécies e em número de indivíduos de acordo com a classificação ecológica.

Table 3. Frequency of occurence, contribution in terms of species number and abundance, according to ecological classifications.

\begin{tabular}{lccc}
\hline $\begin{array}{c}\text { Classificação } \\
\text { ecológica }\end{array}$ & $\begin{array}{c}\text { Frequência de } \\
\text { ocorrência (\%) }\end{array}$ & $\begin{array}{c}\text { Contribuição em número } \\
\text { de espécies (\%) }\end{array}$ & $\begin{array}{c}\text { Contribuição em número de } \\
\text { indivíduos (\%) }\end{array}$ \\
\hline Residentes & 100 & 16 & 10 \\
Marinhos dependentes & 70 & 42 & 14 \\
Marinhos visitantes & 100 & 42 & 76 \\
\hline
\end{tabular}


Tabela 4. Relação entre as abundâncias relativas das espécies $L$. alexandrei e $L$. jocu com os locais de amostragem, levando-se em consideração as suas diferentes complexidades estruturais.

Table 4. Correlation between relative abundance of L.alexandrei and L. jocu, and the studied sites, taking structural complexties into account.

\begin{tabular}{ccccc}
\hline L. alexandrei & \multicolumn{3}{c}{ Faixas de tamanhos } \\
\cline { 2 - 5 } & $\mathbf{<} \mathbf{~ c m}$ & $\mathbf{5 - 1 0} \mathbf{~ c m}$ & $\mathbf{1 0 - 1 5} \mathbf{~ c m}$ & $\mathbf{1 5} \mathbf{- 2 0} \mathbf{~ c m}$ \\
\hline Estações 1e 2* & $12,3 /$ minuto & $10 /$ minuto & $2,7 /$ minuto & - \\
Estação 3** & $11 /$ minuto & $16 /$ minuto & $4 /$ minuto & - \\
\hline L. jocu & & & & - \\
\hline Estações 1e 2* & $1,3 /$ minuto & $6,3 /$ minuto & $2,3 /$ minuto & $1 /$ minuto \\
Estação 3** & 3/minuto & 9/minuto & $5 /$ minuto & - \\
\hline
\end{tabular}

*Grande complexidade estrutural; **pequena complexidade estrutural.

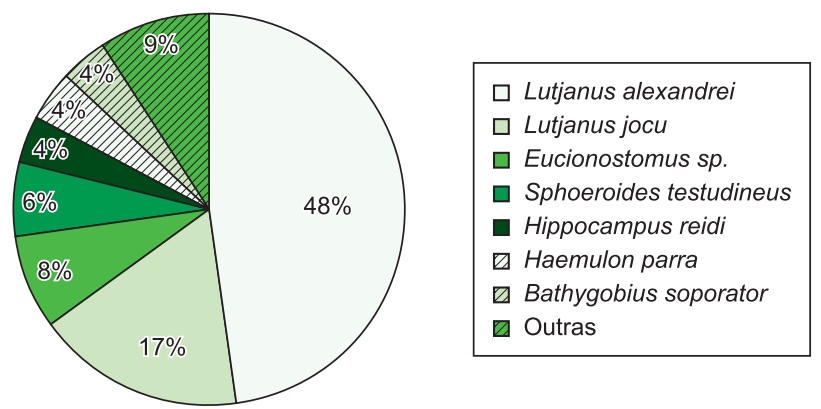

Figura 2. Abundância relativa das espécies.

Figure 2. Relative species abundance.

Nas Antilhas, as espécies Lutjanus apodus (Walbaum 1792) foi dominante em raízes de mangue (Nagelkerken et al. 2000). Vale ressaltar que $L$. apodus é bastante semelhante à espécie recém descrita L. alexandreie identificada incorretamente para o litoral brasileiro, sendo confundida com a primeira (Moura \& Lindeman 2007). Ao que parece, a utilização deste habitat pelo gênero Lutjanus no nordeste brasileiro não é um fato isolado, mas um padrão ecológico de espécies desse gênero. Durante as observações foi verificada a formação de cardumes mistos de indivíduos de $L$. alexandrei e $L$. jocu, com tamanhos inferiores a $20 \mathrm{~cm}$ apenas na estação de coleta mais próxima ao mar (Estação 1). Provavelmente, os indivíduos que habitam áreas próximas ao mar estejam mais vulneráveis a predação, o comportamento de agregação contribui para a proteção dos indivíduos nos seus primeiros estágios de vida. Quando adultos, os indivíduos desta espécie mantém o comportamento gregário em ambientes recifais (Moura \& Lindeman 2007).

Na Ilha Ishigaki, no Japão, L. fulvus (Forster 1801) também utiliza o manguezal como área de berçário (Nakamura et al. 2008). Estes autores observaram que os indivíduos adultos não migram dos recifes para manguezais com a finalidade de alimentação, possivelmente apenas para os eventos reprodutivos.

Algumas espécies que habitam recifes quando adultos utilizam raízes de mangue como habitat nos estágios iniciais de suas vidas (Ley et al. 1999). Foi observado que 68,18\% das espécies avistadas neste trabalho são típicas de ambientes recifais da costa brasileira (Floeter et al. 2003), incluindo-se as duas espécies mais abundantes, o que demonstra a existência de fortes conexões entre esses dois ambientes. Paiva et al. 2009 observou que mais da metade $(51,3 \%)$ das 78 espécies coletadas no Rio Formoso, PE são de origem recifal.

Foram avistados 29 indivíduos de $H$. reidi no ambiente formado pelas raízes de mangue. No Rio Grande do Norte esta espécie apresentou preferência por raízes de Avicennia schaueriana e Rhizophora mangle (Dias \& Rosa 2003), o que corrobora as observações realizadas durante o presente estudo. Tais observações apontam a importância da preservação do manguezal para conservação do cavalo-marinho $H$. reidi, a qual está listada no Anexo II da IN 05/2004 ((Ministério... 2004)), a qual que torna necessário a elaboração de um Plano de Gestão desta espécie pelo IBAMA.
Dentre as espécies observadas neste trabalho $66,7 \%$ são utilizadas no consumo humano (Tabela 2). Em número de indivíduos avistados estas espécies compreendem $83,9 \%$ do total. As espécies do gênero Centropomus e as espécies L. jocu e M. bonaci (Poey 1860) estão entre as mais importantes na pesca extrativa marinha do Ceará (Instituto... 2005). 28,6\% das avistagens correspondem às espécies utilizadas no comércio de peixes ornamentais do Ceará (Monteiro-Neto et al. 2003, Nottingham et al. 2005), demonstrando a importância de raízes de mangue para manutenção da atividade extrativista marinha no Ceará. Os resultados encontrados apontam a necessidade de estudos relacionados ao recrutamento, padrões reprodutivos e padrões tróficos das duas espécies mais representativas.

\section{Agradecimentos}

Agradecemos à Coordenação de Aperfeiçoamento de Pessoal de Nível Superior (CAPES) e à Universidade Federal do Ceará (UFC) pela concessão de bolsas para o primeiro e segundo autor, respectivamente. Agradecemos também ao geólogo Luiz José Cruz Bezerra pela confecção do mapa.

\section{Referências Bibliográficas}

ALBARET, J., SIMIER, M., DARBOE, E.S., ECOUTIN, J., RAFFRAY, J., MORAIS, L.T. 2004. Fish diversity and distribuition in the Gambia Estuary, West Africa, in relation to environmental variables. Aquat Living Resour. 17:35-46.

ALLEN, G.R. 1985. Snappers of the world. An annotated and illustrated catalogue of lutjanid species known to date. FAO species catalogue, Rome.

ARAÚJO, M.E., TEIXEIRA, J.M. \& OLIVEIRA, A.M.E. 2000. Ictiofauna marinha do Estado do Ceará, Brasil: III. Actinopterygii de estuários. Arq. Cienc. Mar. 33:139-142.

ARAÚJO, M.E., TEIXEIRA, J.M. \& OLIVEIRA, A.M.E. 2004. Peixes estuarinos marinhos do Nordeste Brasileiro. Edições UFC, Fortaleza.

CAMPOS, A.A., MONTEIRO, A.Q. \& MONTEIRO-NETO, C. 2003. A zona costeira do Ceará: diagnóstico para a gestão integrada. AQUASIS, Fortaleza.

DIAS, T.L.P. \& ROSA, I.L. 2003. Habitat preferences of a seahorse species, Hippocampus reidi (Teleostei: Syngnathidade) in Brazil. Aqua, J. of Ichtoyol. and Aquatic Biol. 6(4):165-176.

DIEGUES, A.C.S. 1987. Conservação e desenvolvimento sustentado de ecossistemas litorâneos no Brasil. In Simpósio sobre Ecossistemas da Costa Sul e Sudeste Brasileira. Academia de Ciências do Estado de São Paulo, São Paulo 3(54):196-213.

ESKINAZI, A.M. 1970. Lista preliminar dos peixes estuarinos e de Pernambuco e Estados vizinhos (Brasil). Trab. Oceanogr. 9:265-274.

ESKINAZI, A.M. 1972. Peixes do Canal de Santa Cruz, Pernambuco-Brasil. Trab. Oceanogr. 13:283-302.

FLOETER, S.R., GASPARINI, J.L., ROCHA, L.A., FERREIRA, C.E.L., RANGEL, C.A. \& FEITOZA, B.M. 2003. Brazilian reef fish fauna: checklist and remarks. Brazilian Reef Fish Project: www.brazilianreeffish. cjb.net (último acesso em 24/11/2004). 
FREITAS, L.E.L., FEITOSA, C.V. \& ARAÚJO, M.E. 2006. Mangrove oyster (Crassostrea rhizophorae) (GUILDING, 1928) farming areas as artificial reefs for fish: a study in the State of Ceará, Brazil. Braz. J. Oceanogr. 54 (1):31-39.

INSTITUTO BRASILEIRO DO MEIO AMBIENTE E DOS RECURSOS NATURAIS RENOVÁVEIS - IBAMA. 2005. Estatística da pesca 2004 Brasil, grandes regiões e unidades da federação. Brasília, D.F.

LACERDA, L.D., MAIA, L.P., MONTEIRO, L.H.U., SOUZA, G.M., BEZERRA, L.J.C. \& MENEZES, M.O.T. 2006. Manguezais do Nordeste e mudanças ambientais. Cienc. Hoje 29(229):24-29.

LAEGDSGAARD, P. \& JOHNSON, C. 2001. Why do juvenile fish utilise mangrove habitats? J. Exp. Mar. Biol. Ecol. 257:229-253.

LEY, J.A., MCIVOR, C.C. \& MONTAGUE, C.L. 1999. Fishes in mangrove prop-root habitats of Northeastern Florida Bay: dinstict assemblages across an estuarine gradient. Estuar. Coast Shelf S. 48:701-723.

LOEBMANN, D. \& VIEIRA, J.P. 2005. Distribuição espacial e abundância das assembléias de peixe no Parque Nacional da Lagoa do Peixe, Rio Grande do Sul, Brasil. Rev. Bras. Zool. 22 (3):667-675.

LOWE-MACCONNELL, R.H. 1999. Estudos ecológicos de comunidades de peixes tropicais. Editora da Universidade de São Paulo, São Paulo.

MENEZES, N.A. \& FIGUEIREDO, J.L. 1980. Manual de peixes marinhos do Sudeste do Brasil: IV Teleostei (3). Museu de Zoologia; Universidade de São Paulo, São Paulo.

MINISTÉRIO DO MEIO AMBIENTE - MMA. 2004. Instrução normativa $\mathrm{n}^{\circ}$ 5, de 21 de maio de 2004. Diário Oficial da União - seção $1, \mathrm{n}^{\circ} 102$, sexta-feira, 28 de maio de 2004.

MONTEIRO-NETO, C., CUNHA, F.E.A., NOTTINCHAM, M.C., BARROS, M.G.L., ARAÚJO, M.E. \& ROSA, I.L. 2003. Analysis of the marine ornamental fish trade at Ceará State, Northeast Brazil. Biodivers. Conserv. 12:1287-1295.

MORTON, R.M. 1990. Community structure, density and standing crop of fishes in a subtropical Australian mangrove area. Mar. Biol. 105:385-394.

MOURA, R.L., FIGUEIREDO, J.L. \& SAZIMA, I. 2001. A new parrofish (Scaridade) from Brazil, and revalidation of Sparisoma amplum (Ranzani, 1842), Sparisoma frondosum (Agassiz, 1831), Sparisoma axillare (Steindachner, 1878) and Scarus trispinosus (Valenciennes, 1840). B. Mar. Sci. 68(3):505-524.

MOURA, R.L. \& LINDEMAN, K.C. 2007. A new species of snapper (Perciformes: Lutjanidae) from Brazil, with comments on the distribution of Lutjanus griseus and L. apodus. Zootaxa 1422:31-43.

MULLIN, S.J. 1995. Estuarine fish populations among red mangrove prop roots of small overwash islands. Wetlands 15:324-329.

NAKAMURA, Y., HORINOUCHI, M., SHIBUNO, T., TANAKA, Y., MIYAJIMA, T., KOIKE, I., KUROKURA, H. \& SANO, M. 2008. Evidence of ontogenetic migration from mangroves to coral reefs by black-tail snapper Lutjanus fulvus: stable isotope approach. Mar. EcolProg. Ser. 355:257-266.
NAGELKERKEN, I., VELDE, G., GORISSEN, M.W., MEIJER, G.J., HOF, T. \& HARTOG, C. 2000. Importance of mangroves, seagrass beds and the shallow coral reef as a nursery for important coral reef fishes, using a visual census technique. Estuar. Coast Shelf S. 51:31-44.

NELSON, J.S. 2006. Fishes of the world. New York, John Wiley \& Sons, 600p.

NOTTINGHAM, M.C., BARRETO, L.M., ARAÚJO, M.E., MONTEIRONETO, C., CUNHA, F.E.A., ROSA, I.M.L. \& ALENCAR, C.A.G. 2005. A explotação de peixes ornamentais marinhos no Estado do Ceará, Brasil: captura, manutenção nas empresas e exportação. Bol. Tec. Cient. CEPENE. 13(1):53-73.

ODUM, E.P. 1988. Ecologia. Guanabara, Rio de Janeiro.

OLIVEIRA, A.M.E. 1972. Peixes estuarinos do Nordeste oriental brasileiro. Arq. Cienc. Mar. 12(1):35-41.

OLIVEIRA, A.M.E. 1974. Ictiofauna das águas estuarinas do Rio Parnaíba (Brasil). Arq. Cienc. Mar. 14(1):41-45.

OLIVEIRA, A.M.E. 1976. Composição e distribuição da ictiofauna nas águas estuarinas do rio Jaguaribe (Ceará - Brasil). Arq. Cienc. Mar. 16(1):9-18.

OLIVEIRA, A.M.E., IRVING, M.A. \& LIMA, H.H. 1988. Aspectos bioecológicos do estuário do rio Pacoti, Ceará, Brasil. Arq. Cienc. Mar. 16:91-100.

PAIVA, A.C.G., CHAVES, P.T.C. \& ARAÚJO, M.E. 2008. Estrutura e organização trófica da ictiofauna de águas rasas em um estuário tropical. Rev. Bras. Zool. 25(4):647-661.

PAIVA, A.C.G., LIMA, M.F.V., SOUZA, J.R.B., ARAÚJO, M.E. 2009. Spatial distribuition of the estuarine ichthyofauna of the Rio Formoso (Pernambuco, Brazil), with emphasis on reef fish. Zoologia 26 (2):266-278.

THAYER, G.W., COLBY, D.R. \& HETTLER, W.F. 1987. Utilization of the red mangrove prop root habitat by fishes in south Florida. Mar Ecol-Prog Ser. 35:25-38.

VANCE, D.J., HAYWOOD, M.D.E., HEALES, D.S., KENYON, R.A., LONERAGAN, N.R. \& PENDREY, R.C. 1996. How far do prawns and fish move into magroves? Distribution of juvenile banana prawns Penaeus merguiensis and fish in a tropical mangrove forest in norther Australia. Mar. Ecol-Prog. Ser. 131:115-124.

VASCONCELOS-FILHO, A.L. \& OLIVEIRA, A.M.E. 1999. Composição e ecologia da ictiofauna do Canal de Santa Cruz (Itamaracá - PE, Brasil). Trab. Oceanogr. 27(1):101-113.

ZEE. 2005a. Zoneamento Ecológico e Econômico (ZEE) da Zona Costeira do Estado do Ceará: caracterização das condições físico-químicas dos estuários do estado do Ceará. Fortaleza, Governo do Estado do Ceará, Universidade Federal do Ceará. SEMACE/LABOMAR. http://www. semace.ce.gov.br/ (último acesso em 24/07/2010).

ZEE. 2005b. Zoneamento Ecológico e Econômico (ZEE) da Zona Costeira do Estado do Ceará: ictiofauna dos estuários do estado do Ceará. Fortaleza, Governo do Estado do Ceará, Universidade Federal do Ceará. SEMACE/LABOMAR. http://www.semace.ce.gov.br/ (último acesso em 24/07/2010). 